\title{
Desenvolvimento financeiro e decisões \\ de investimento das firmas brasileiras
}

\author{
Aquiles Elie Guimarães Kalatzis* \\ Fernanda de Castro**
}

\begin{abstract}
RESUMO - Este artigo analisa os efeitos do desenvolvimento financeiro e das restrições financeiras nas decisões de investimento de 659 firmas brasileiras classificadas por grau de restrição financeira no período de 1998 a 2006. A análise é feita a partir de uma versão do modelo acelerador do investimento estimado pelo método dos momentos generalizados (GMM). Os resultados indicam que o desenvolvimento financeiro possui um importante papel nas decisões de investimento das firmas brasileiras, ao reduzir o custo de captação de recursos externos e ao melhorar a alocação de capital na economia. Maiores níveis de desenvolvimento financeiro também se apresentam associados a uma redução no grau de restrição financeira de firmas identificadas como financeiramente restritas, ao diminuir sua dependência por fundos internos como fonte de financiamento.
\end{abstract}

Palavras-chave: Decisões de investimento. Desenvolvimento financeiro. Restrição financeira.

\section{INTRODUÇÃO}

A identificação do comportamento das decisões de investimento empresarial tem sido um tema de grande importância na literatura da teoria do investimento, não apenas ao que se refere às decisões de orçamento de capital no âmbito da firma, mas devido à sua relação com o desenvolvimento financeiro e crescimento econômico. De acordo com a teoria econômica, o desenvolvimento dos mercados financeiros pode proporcionar reduções nos custos de transação e informação, influenciando a queda das taxas de captação de recursos e, consequentemente, exercendo influência sobre as decisões de investimento das firmas.

A importância do desenvolvimento do setor financeiro já era destacada por Schumpeter (1911), no entanto, apenas recentemente alguns estudos tem procurado destacar a relação entre o desenvolvimento dos mercados financeiros e a economia real.

A partir do trabalho de Modigliani e Miller (1958), essa relação sofreu avanços significativos ao propor que em um mercado de capitais perfeito a estrutura financeira da firma tornase irrelevante na tomada de decisões reais. No entanto, essa perspectiva foi bastante questionada

\footnotetext{
* Doutor em Economia pela Universidade de São Paulo. É professor associado do Departamento de Engenharia de Produção da Escola de Engenharia de São Carlos, Universidade de São Paulo. Endereço eletrônico: aquiles@ sc.usp.br.

** Mestre em Engenharia de Produção pela Escola de Engenharia de São Carlos, Universidade de São Paulo. É doutoranda em Engenharia de Produção pela Escola de Engenharia de São Carlos, Universidade de São Paulo. Endereço eletrônico: fernandacastro@usp.br.
} 
por diversos trabalhos empíricos, sobretudo por Fazzari, Hubbard e Petersen (1988), os quais apontam para a existência de mercados imperfeitos em virtude dos problemas de informação. Como consequência, surgiria um hiato entre o custo de recursos externos e o custo do capital próprio, tornando as firmas mais dependentes dos ganhos retidos para a realização de novos investimentos, aumentando seu grau de restrição financeira.

Embora muitos estudos na literatura procurem evidenciar o impacto das restrições financeiras no comportamento das firmas e outros ressaltem a importância do desenvolvimento financeiro para o crescimento econômico, ainda são poucos os trabalhos que incorporam ambas as literaturas em estudos sobre os investimentos empresariais. Entre tais estudos, destaca-se o trabalho de Love (2003), que analisa o papel do desenvolvimento financeiro nas decisões de investimento considerando seu efeito sobre os problemas de restrição financeira das firmas. Como resultado, esse estudo sugere que o nível de restrição financeira das firmas reduz-se para maiores níveis de desenvolvimento dos mercados financeiros. Resultado semelhante foi encontrado por Islam e Mozumdar (2007), e em um estudo mais recente Baum, Schäfer e Talavera (2011) encontram que tanto a estrutura financeira de um país quanto o seu nível de desenvolvimento financeiro possuem um importante papel na flexibilização das restrições financeiras das firmas.

Nesse sentido, este trabalho analisa os efeitos do desenvolvimento financeiro e das restrições financeiras nas decisões de investimento da firma a partir de informações de 659 empresas brasileiras no período de 1998 a 2006. Um aspecto interessante deste trabalho é a sua contribuição à discussão a respeito dos efeitos do desenvolvimento financeiro sobre as decisões de investimento e restrições financeiras das firmas brasileiras, até hoje não examinada na literatura nacional. A investigação é realizada dentro de um contexto teórico e aplicado a partir de um modelo econométrico com dados longitudinais, sendo considerada na estimação dos parâmetros uma versão do modelo acelerador do investimento, o qual é estimado via GMM devido seu caráter dinâmico e à presença do problema de endogeneidade.

Este artigo está estruturado em quatro seções, considerando esta introdução. A Seção 2 descreve os dados e o método proposto neste estudo. A Seção 3 analisa os principais resultados das estimações. Por fim, são apresentadas as conclusões desta pesquisa.

\section{DADOS E MÉTODO}

Este trabalho utiliza informações de 659 empresas brasileiras privadas de capital aberto e fechado no período de 1998 a 2006. A fonte provedora de dados foi o IBRE - Instituto Brasileiro de Economia, da Fundação Getúlio Vargas. Todas as informações constantes no ban- 
co de dados foram deflacionadas segundo o Índice Geral de Preços - Disponibilidade Interna (IGP-DI), tomando 2006 como ano base.

Além de informações microeconômicas, coletadas a partir do balanço contábil das firmas da amostra, este estudo também fez uso de variáveis agregadas e de informações do mercado de crédito e de capitais do Brasil, obtidas a partir dos sites do Ipeadata e do Banco Central do Brasil. Também foram utilizadas informações obtidas a partir do Financial Structure Database de Beck, Demirgüç-Kunt e Levine (2000), atualizado em abril de 2010.

O modelo proposto neste trabalho para analisar os efeitos do desenvolvimento financeiro e das restrições financeiras nas decisões de investimento da firma foi uma versão do modelo acelerador do investimento dado por:

$$
\begin{gathered}
{\left[\frac{I}{K_{t-1}}\right]_{i t}=\alpha_{i}+\beta_{1}\left[\frac{I}{K_{t-1}}\right]_{i, t-1}+\beta_{2}\left[\frac{I}{K_{t-1}}\right]_{i, t-1}^{2}+\beta_{3}\left[\frac{F C}{K_{t-1}}\right]_{i t}+\beta_{4}\left[\frac{V}{K_{t-1}}\right]_{i t}+\beta_{5}\left[\frac{D}{K_{t-1}}\right]_{i t}+} \\
+\beta_{6} \log (P I B)_{t}+\beta_{7} F D_{t}+\beta_{8}\left[\frac{F C}{K_{t-1}}\right]_{i t} . F D_{t}+\varepsilon_{i t}
\end{gathered}
$$

onde $t$ é o ano; $i$ é a firma; $\alpha_{i}$ é o efeito fixo; $K_{i t}$ é o estoque de capital em ativos fixos (imobilizado); $I_{i t}$ é o investimento da firma, definido como $K_{i t}-K_{i t 1} ; F C_{i t}$ é o fluxo de caixa, dado pela soma do lucro líquido mais depreciação; $V_{i t}$ indica vendas; $D_{i t}$ é a dívida; $\log (\text { PIB })_{t}$ é o logaritmo do PIB; FD é a variável de desenvolvimento financeiro, dada pelo logaritmo da soma do percentual do crédito total alocado ao setor privado em relação ao PIB mais o percentual da capitalização no mercado acionário em relação ao PIB $^{1}$; e $\varepsilon_{i t}$ é o termo de erro.

A presença da variável $\left(I / K_{t-1}\right)_{i, t-1}$ como explicativa no modelo de investimento objetiva proporcionar um caráter dinâmico ao mesmo. A variável quadrática reflete a presença de um comportamento não linear no processo de ajustamento do estoque de capital. A variável (FC/ $\left.K_{t-1}\right)_{i t}$ considera o efeito de possíveis restrições financeiras no comportamento investidor das firmas, embora também possa representar potencial de rentabilidade futura. A variável $\left(V / K_{t-1}\right)_{i t}$ considera o papel da taxa de mudança no nível de vendas ou produção. A introdução da variável $\left(D / K_{t-1}\right)_{i t}$ deve-se aos benefícios tributários da dívida e ao fato de que uma maior alavancagem pode aumentar o valor da firma. A variável $\log (P I B)_{t}$ captura o efeito do crescimento econômico nas decisões de investimento empresariais. Considerando que um maior nível de desenvol-

1 Embora não exista na literatura uma única variável de desenvolvimento financeiro, a variável empregada neste trabalho é comumente utilizada em estudos internacionais para representar o nível de desenvolvimento financeiro de um país. Para uma melhor definição desta e de outras variáveis de desenvolvimento financeiro, ver Beck et al. (2001). 
vimento financeiro pode vir acompanhado por um maior crescimento da economia, a inclusão desta variável é necessária a fim de garantir que a variável de desenvolvimento financeiro esteja realmente captando o efeito de um maior nível de desenvolvimento do setor financeiro nas decisões de investimento e não esteja meramente refletindo o efeito do crescimento econômico nas decisões empresariais. Já a variável $F D_{t}$ tem como objetivo determinar se maiores níveis de desenvolvimento financeiro estão associados a maiores taxas de investimento, o que implica $\beta_{7}>0$. Por fim, a variável de interação $\left(F C / K_{t-1}\right)_{i t} . F D_{t}$ tem como objetivo captar o efeito do desenvolvimento financeiro sobre as restrições financeiras, de forma que se espera que para maiores níveis de desenvolvimento financeiro exista uma menor dependência dos investimentos por variáveis de liquidez interna, reduzindo o grau de restrição financeira das firmas. Isso implicaria $\beta_{8}<0$.

Para considerar o aspecto dinâmico e o problema de endogeneidade do modelo descrito pela Equação (1), o mesmo foi estimado pelo método GMM, sugerido por Arellano e Bond (1991), que consiste em tirar a primeira diferença e utilizar instrumentos adequados para a variável endógena. Neste estudo, foram empregadas a segunda e a terceira defasagem da variável dependente em nível como instrumentos para a variável explicativa endógena. Assumiu-se também a variável $\left(F C / K_{t-1}\right)_{i t}$ como fracamente exógena, utilizando como instrumentos suas três primeiras defasagens em nível. O estimador GMM aqui empregado é obtido em duas etapas (two-step GMM estimator), onde na primeira etapa (first step) obtém-se a matriz de ponderação ótima, a qual é utilizada na segunda etapa do processo de estimação.

A fim de se considerar a heterogeneidade existente entre as diferentes firmas, a amostra foi dividida em dois grupos de firmas, classificadas como financeiramente restritas e não restritas de acordo com o índice KZ. Essa classificação possibilitou tornar a amostra mais homogênea dentro de cada grupo amostral, permitindo uma melhor análise a respeito do diferente comportamento investidor entre firmas com diferentes graus de restrição financeira. $\mathrm{O}$ índice $K Z$ aqui utilizado é dado por ${ }^{2}$ :

$$
K Z_{i t}=-\left[1,001009 \frac{F C}{K_{t-1}}\right]_{i t}+\left[3,139193 \frac{D}{\text { TotCap }}\right]_{i t}-\left[1,314759 \frac{\text { Caixa }}{K_{t-1}}\right]
$$

onde té ano; $i$ é a firma; $K_{i t}$ é o estoque de capital em ativos fixos (imobilizado); $F C_{i t}$ é o fluxo $2 \mathrm{O}$ índice $K Z$ foi desenvolvido por Lamont, Polk e Saá-Requejo (2001) a partir do trabalho de Kaplan e Zingales (1997). No entanto, devido à indisponibilidade de algumas variáveis no banco de dados utilizado neste estudo, o índice $K Z$ aqui proposto considera apenas três das cinco variáveis as quais compõem o índice original. Apesar do número reduzido de variáveis utilizadas na construção do índice $K Z$, o mesmo mostrou-se uma boa medida de restrição financeira visto que consegue captar a essência na classificação das firmas conforme os graus de restrição financeira das mesmas. 
de caixa, dado pela soma do lucro líquido mais depreciação; $D_{i t}$ é a variável dívida; TotCap ${ }_{i t}$ é o capital total, definido como a soma da dívida mais o patrimônio líquido; e Caixa $a_{i t}$ é caixa, definido como a variável disponível do balanço da firma.

Dessa forma, a fim de classificar as firmas em dois grupos distintos, a amostra foi dividida em tercis de acordo com os valores assumidos pelo índice $K Z$ e firmas que pertenciam ao tercil mais alto foram classificadas como financeiramente restritas e firmas que pertenciam ao tercil mais baixo foram classificadas como não restritas financeiramente.

\section{RESULTADOS}

Essa seção analisa os principais resultados econômicos estimados a partir do modelo descrito pela Equação (1). A Tabela 1 apresenta as médias das principais variáveis financeiras para cada grupo de firmas. Nota-se que firmas consideradas financeiramente restritas apresentam valores substancialmente menores para as variáveis investimento, fluxo de caixa, vendas, capital de giro e lucro líquido, todas como razão do estoque de capital. Os piores indicadores de variáveis de liquidez e menor lucratividade para esse grupo de firmas podem estar sinalizando uma maior sensibilidade do investimento ao fluxo de caixa e maior restrição financeira. No entanto, caso a variável fluxo de caixa esteja sinalizando potencial de rentabilidade futura, esperase que firmas não restritas financeiramente apresentem maior sensibilidade do investimento ao fluxo de caixa que firmas restritas financeiramente.

TABELA 1 - CARACTERÍSTICAS FINANCEIRAS DAS FIRMAS: VALORES MÉDIOS E DESVIO PADRÃO PARA FIRMAS CLASSIFICADAS POR GRAU DE RESTRIÇÃO FINANCEIRA DE ACORDO COM O ÍNDICE $K Z$

\begin{tabular}{|c|c|c|c|c|c|c|}
\hline \multirow{2}{*}{ Indicadores } & \multicolumn{2}{|c|}{ Amostra total } & \multicolumn{2}{|c|}{ Firmas não restritas } & \multicolumn{2}{|c|}{ Firmas restritas } \\
\hline & Média & Desv. pad. & Média & Desv. pad. & Média & Desv. pad. \\
\hline Investimento $/ K_{t-1}$ & 0,0412 & 0,6649 & 0,0943 & 0,9098 & $-0,0007$ & 0,4879 \\
\hline Fluxo de caixa $/ K_{t-1}^{t-1}$ & 0,5198 & 2,9974 & 1,4591 & 4,3908 & $-0,1419$ & 2,4898 \\
\hline Vendas $/ K_{t-1}$ & 5,8306 & 11,6374 & 10,2902 & 17,7500 & 3,5038 & 6,2561 \\
\hline Dívida $/ K_{t-1}^{t-1}$ & 1,1482 & 4,4141 & 1,5055 & 5,4626 & 1,3727 & 4,9986 \\
\hline Capital de giro $/ K_{t}$ & 1,6903 & 7,9109 & 4,0281 & 12,5755 & 0,2015 & 2,5869 \\
\hline Lucro líquido $/ K_{t-1}^{t-1}$ & 0,3949 & 2,9935 & 1,3277 & 4,3807 & $-0,2619$ & 2,4987 \\
\hline Ativo total & $8,37 e+08$ & $2,66 \mathrm{e}+09$ & $5,31 e+08$ & $1,47 e+09$ & $1,19 \mathrm{e}+09$ & $2,80 \mathrm{e}+09$ \\
\hline Dívida/Patrimônio líquido & 0,7887 & 7,8660 & 0,1334 & 0,4371 & 2,0518 & 14,1210 \\
\hline Número de observações & & 5.931 & & 1.757 & & 1.757 \\
\hline
\end{tabular}

FONTE: Cálculo dos autores.

A Tabela 2 apresenta os parâmetros estimados para o modelo dado pela Equação (1). Os testes de Sargan e de autocorrelação confirmam a validade dos modelos estimados. Pela Tabela 2, é possível observar que a variável $\left(F C / K_{t-1}\right)_{i t}$ apresenta-se positiva nas duas especificações do modelo estimado, porém, significante apenas para firmas classificadas como financeiramente 
restritas. Esse resultado, quando associado à baixa lucratividade deste grupo de firmas, permite a conclusão de que o fluxo de caixa está sinalizando a presença de restrição financeira devido a uma maior dependência de recursos internos para o empreendimento de novos investimentos no caso de firmas classificadas como financeiramente restritas.

TABELA 2 - ESTIMAÇÃO DOS PARÂMETROS DO MODELO DE INVESTIMENTO PELO MÉTODO GMM COM FIRMAS CLASSIFICADAS POR GRAU DE RESTRIÇÃO FINANCEIRA DE ACORDO COM O ÍNDICE $K Z$

\begin{tabular}{|c|c|c|c|c|c|}
\hline \multirow{2}{*}{\multicolumn{2}{|c|}{ Variáveis }} & \multicolumn{2}{|c|}{ (1) } & \multicolumn{2}{|c|}{ (2) } \\
\hline & & \multirow{3}{*}{$\begin{array}{c}\begin{array}{c}\text { Firmas não } \\
\text { restritas }\end{array} \\
-0,0116 \\
(0,0450)\end{array}$} & \multirow{2}{*}{$\frac{\text { Firmas restritas }}{0,4094^{* * *}}$} & \multirow{3}{*}{$\begin{array}{c}\begin{array}{l}\text { Firmas não } \\
\text { restritas }\end{array} \\
-0,0121 \\
(0,0458)\end{array}$} & \multirow{2}{*}{$\begin{array}{r}\text { Firmas restritas } \\
0,3967^{* * *}\end{array}$} \\
\hline \multirow{4}{*}{$\left(I / K_{t-1}\right)_{i t}$} & LD & & & & \\
\hline & & & $(0,0420)$ & & $(0,0420)$ \\
\hline & $\mathrm{L} 2 \mathrm{D}$ & $-0,0323 * * *$ & $-0,0887 * * *$ & $-0,0319 * * *$ & $-0,0897 * * *$ \\
\hline & & $(0,0022)$ & $(0,0081)$ & $(0,0023)$ & $(0,0085)$ \\
\hline \multirow{2}{*}{$\left(I / K_{t-1}\right)_{i t-1}^{2}$} & D1 & $-0,0055$ & $-0,1311 * * *$ & $-0,0055$ & $-0,1279 * * *$ \\
\hline & & $(0,0049)$ & $(0,0102)$ & $(0,0050)$ & $(0,0101)$ \\
\hline \multirow{2}{*}{$\left(F C / K_{t-1}\right)_{i t}$} & D1 & 0,1374 & $0,1603^{* * *}$ & 0,1243 & $0,1603^{* * *}$ \\
\hline & & $(0,1078)$ & $(0,0476)$ & $(0,1049)$ & $(0,0528)$ \\
\hline \multirow[t]{2}{*}{$\left(V / K_{t-1}\right)_{i t}$} & D1 & $0,0082^{* * *}$ & $0,0460 * * *$ & $0,0089 * * *$ & $0,0468^{* * *}$ \\
\hline & & $(0,0019)$ & $(0,0063)$ & $(0,0019)$ & $(0,0064)$ \\
\hline \multirow{2}{*}{$\left(D / K_{t-1}\right)_{i t}$} & D1 & $0,0426^{* *}$ & $0,0202^{* * *}$ & $0,0401 * *$ & $0,0209 * * *$ \\
\hline & & $(0,0171)$ & $(0,0062)$ & $(0,0172)$ & $(0,0063)$ \\
\hline \multirow[t]{2}{*}{$\log (P I B)_{t}$} & D1 & & & $0,7284 * * *$ & 0,2554 \\
\hline & & & & $(0,2494)$ & $(0,2713)$ \\
\hline \multirow[t]{2}{*}{$(F D)_{t}$} & D1 & $0,5175^{* * *}$ & $0,4058^{* * *}$ & $0,3428 * * *$ & $0,3386 * *$ \\
\hline & & $(0,1349)$ & $(0,1151)$ & $(0,1274)$ & $(0,1371)$ \\
\hline \multirow{2}{*}{$\left(F C / K_{t-1}\right)_{i t} \cdot(F D)_{t}$} & D1 & $-0,0250$ & $-0,0387 * * *$ & $-0,0226$ & $-0,0388 * * *$ \\
\hline & & $(0,0261)$ & $(0,0117)$ & $(0,0255)$ & $(0,0129)$ \\
\hline \multirow{2}{*}{\multicolumn{2}{|c|}{ Constante }} & $0,0350 * * *$ & $-0,0533 * * *$ & $0,0279 * *$ & $-0,0546^{* * *}$ \\
\hline & & $(0,0117)$ & $(0,0116)$ & $(0,0113)$ & $(0,0115)$ \\
\hline \multicolumn{2}{|l|}{ Número de firmas } & 365 & 366 & 365 & 366 \\
\hline \multicolumn{2}{|c|}{ Número de observações } & 1.182 & 1.062 & 1.182 & 1.062 \\
\hline \multicolumn{6}{|c|}{ Testes de especificação ( $\not$-valores) } \\
\hline (a) Teste de Sarga & & 0,3194 & 0,1527 & 0,3697 & 0,1452 \\
\hline \multicolumn{6}{|c|}{ (b) Teste de autocorrelação } \\
\hline Primeir & & 0,1193 & 0,0232 & 0,1229 & 0,0263 \\
\hline Segunda & & 0,5691 & 0,7170 & 0,5984 & 0,7234 \\
\hline Terceira & & 0,5438 & 0,6884 & 0,6693 & 0,7065 \\
\hline
\end{tabular}

FONTE: Cálculo dos autores.

NOTA: O modelo estimado é dado pela Equação (1); a variável dependente é $\left(I / K_{t-1}\right)_{i t}$ e LD, L2D e D1 representam diferença defasada, segunda diferença defasada e primeira diferença, respectivamente; os erros-padrão estão reportados entre parênteses; os símbolos $\left(^{* * *}\right)$ e $\left(^{* *}\right)$ indicam significância estatística em $1 \%$ e $5 \%$.

O coeficiente da variável $\left(V / K_{t-1}\right)_{i t}$ apresenta-se significante e positivo para ambos os grupos de firmas, confirmando o princípio do modelo acelerador inicial, em que o estoque de bens de capital é proporcional a mudanças no seu nível de vendas ou produção. A variável $\left(D / K_{t-1}\right)_{i t}$ também se apresenta significante e positiva para ambos os grupos de firmas, porém, com maior valor para as firmas classificadas como não restritas financeiramente. Isso ocorre, provavelmente, devido ao fato de essas firmas serem de menor porte que firmas financeiramen- 
te restritas, implicando em uma menor disponibilidade de colaterais para obtenção de recursos externos, o que pode acentuar o parâmetro da variável dívida para esse grupo de firmas.

Pela Tabela 2 também é possível verificar que na ausência da variável $\log (P I B)_{t}$ a variável de desenvolvimento financeiro, $F D_{t}$, apresenta-se positiva e significante na explicação do investimento das firmas. No entanto, quando inserida a variável $\log (P I B)_{t}$ no modelo estimado observa-se que, embora a magnitude do parâmetro da variável $F D_{t}$ se reduza, a mesma ainda permanece positiva e significante para ambos os grupos de firmas, confirmando o importante papel do desenvolvimento financeiro nas decisões de investimento das firmas brasileiras. Considerando que maiores níveis de desenvolvimento financeiro estão associados a uma maior disponibilidade de crédito, menores custos de captação de recursos externos far-se-iam presentes. Isso geraria um incentivo às firmas investirem mais diante da possibilidade de captação de recursos externos a um custo mais baixo. Ademais, os efeitos do desenvolvimento financeiro sobre os investimentos empresariais também se refletiriam em uma melhora na alocação de capital da economia, ao permitir que as firmas brasileiras invistam de acordo com suas oportunidades de crescimento. Já o sinal negativo associado à variável de interação $\left(F C / K_{t-1}\right)_{i t} . F D_{t}$ e sua significância apenas para firmas identificadas como financeiramente restritas sugere que o desenvolvimento financeiro reduz o grau de restrição financeira desse grupo de firmas ao diminuir a dependência de seus investimentos por fontes internas de financiamento. Resultado semelhante foi encontrado por Love (2003).

Dessa forma, os principais resultados econômicos encontrados neste trabalho ressaltam o importante papel do desenvolvimento financeiro nas decisões de investimento das firmas brasileiras, evidenciando a grande importância das políticas voltadas a um maior desenvolvimento financeiro nas decisões microeconômicas.

\section{CONCLUSÕES}

Este artigo analisou os efeitos do desenvolvimento financeiro e das restrições financeiras nas decisões de investimento da firma a partir de uma amostra de 659 firmas brasileiras no período de 1998 a 2006. A fim de atingir o objetivo proposto, este trabalho classificou as firmas como financeiramente restritas e não restritas de acordo com o índice $K Z$ e utilizou uma versão do modelo acelerador do investimento estimado pelo método dos momentos generalizados (GMM). Neste estudo, a baixa rentabilidade das firmas classificadas como financeiramente restritas e o impacto positivo e significante do fluxo de caixa sobre as decisões de investimento apenas para este grupo de firmas tornaram possível a identificação da variável fluxo de caixa como proxy para restrição financeira. Os resultados também apontaram para um impacto po- 
sitivo do nível de desenvolvimento financeiro do país nas decisões de investimento das firmas brasileiras. Esse processo ocorre visto que maiores níveis de desenvolvimento financeiro estariam associados a menores custos de captação de recursos externos e a uma melhor alocação de capital na economia. Evidências também sugerem um importante papel do desenvolvimento financeiro para firmas consideradas financeiramente restritas ao diminuir sua dependência por fundos internos como fonte de financiamento, reduzindo seu grau de restrição financeira.

\section{REFERÊNCIAS}

ARELLANO, M.; BOND, S. Some tests of specification for panel data: Monte Carlo evidence and an application to employment equations. The Review of Economic Studies, v. 58, n. 2, p. 277-297, 1991.

BAUM, C. F.; SCHÄFER, D.; TALAVERA, O. The impact of the financial system's structure on firms' financial constraints. Journal of International Money and Finance, v. 30, p. 678691, 2011.

BECK, T.; DEMIRGÜÇ-KUNT, A.; LEVINE, R. A new database on financial development and structure. World Bank Economic Review, v. 14, p. 597-605, 2000.

BECK, T. et al. Financial structure and economic development: firm, industry and country evidence. In: DEMIRGÜÇ-KUNT, A.; LEVINE, R. Financial structure and economic growth: a cross-country comparison of banks, markets, and development. Cambridge: MIT Press, 2001, p. 189-242.

FAZZARI, S.; HUBBARD, G.; PETERSEN, B. Financing constraints and corporate investment. Brookings Papers on Economic Activity, n. 1, p. 141-206, 1988.

ISLAM, S. S.; MOZUMDAR, A. Financial market development and the importance of internal cash: evidence from international data. Journal of Banking and Finance, v. 31, p. 641-658, 2007.

KAPLAN, S.; ZINGALES, L. Do investment-cash flow sensitivities provide useful measures of financing constraints? The Quartely Journal of Economics, v. 112, p. 169-215, 1997.

LAMONT, O.; POLK, C.; SAÁ-REQUEJO, J. Financial constraints and stock returns. The Review of Financial Studies, v. 14, n. 2, p. 529-554, 2001.

LOVE, I. Financial development and financing constraints: international evidence from the structural investment model. The Review of Financial Studies, v. 16, n. 3, p. 765-791, 2003.

MODIGLIANI, F.; MILLER, M. The cost of capital, corporation finance and the theory of investment. American Economic Review, v. 48, n. 3, p. 261-297, 1958.

SCHUMPETER, J. A. A theory of economic development. Cambridge: Harvard University Press, 1911. 